\title{
Thermofield Dynamics and Casimir Effect for Fermions
}

\author{
H. Queiroz ${ }^{a}$, J. C. da Silva ${ }^{a, b}$, F. C. Khanna ${ }^{c, d}$, J. \\ M. C. Malbouisson ${ }^{a, c, *}$, M. Revzen ${ }^{c, e}$, A. E. Santana ${ }^{f}$ \\ ${ }^{a}$ Instituto de Física, Universidade Federal da Bahia, \\ Campus de Ondina, 40210-340, Salvador, BA, Brazil \\ ${ }^{b}$ Centro Federal de Educação Tecnológica da Bahia, \\ Rua Emídio Santos, 40000-900, Salvador, BA, Brazil \\ ${ }^{c}$ Physics Department, Theoretical Physics Institute, \\ University of Alberta, Edmonton, AB T6G 2J1, Canada \\ ${ }^{d}$ TRIUMF, 4004, Westbrook mall, Vancouver, BC V6T 2A3, Canada \\ ${ }^{e}$ Department of Physics, Technion - Institute of Technology, Haifa, 32000, Israel \\ ${ }^{f}$ Instituto de Física, Universidade de Brasília, 70910-900, Brasília, DF, Brazil
}

\begin{abstract}
A generalization of the Bogoliubov transformation is developed to describe a space compactified fermionic field. The method is the fermionic counterpart of the formalism introduced earlier for bosons (J. C. da Silva, A. Matos Neto, F.C. Khanna and A.E. Santana, Phys. Rev. A 66 (2002) 052101), and is based on the thermofield dynamics approach. We analyze the energy-momentum tensor for the Casimir effect of a free massless fermion field in a $d$-dimensional box at finite temperature. As a particular case the Casimir energy and pressure for the field confined in a 3-dimensional parallelepiped box are calculated. It is found that the attractive or repulsive nature of the Casimir pressure on opposite faces changes depending on the relative magnitude of the edges. We also determine the temperature at which the Casimir pressure in a cubic box changes sign and estimate its value when the edge of the cube is of the order of the confining lengths for baryons.
\end{abstract}

PACS: 03.70.+k; 11.10.Wx

Keywords: Fermionic Casimir effect; finite temperature

\footnotetext{
* Corresponding author.

E-mail addresses: hebe@fis.ufba.br (H. Queiroz), jcsilva@cefetba.br (J.C. da Silva), khanna@phys.ualberta.ca (F.C. Khanna), jmalbou@phys.ualberta.ca (J.M.C. Malbouisson), revzen@physics.technion.ac.il (M. Revzen), asantana@fis.unb.br (A.E. Santana).
} 


\section{INTRODUCTION}

In order to calculate the partition function for a quantum field, the Matsubara prescription is equivalent to a path-integral calculated on $R^{D-1} \times S^{1}$, where $S^{1}$ is a circle of circumference $\beta=1 / T$. This non-trivial result was demonstrated by Polchinski [1] at the one-loop level and has been assumed in higher order [2]. Under this perspective, taking Euclidian theories, the Dolan and Jackiw approach [3] can be a useful method to perform calculations for confined (bosonic or fermionic) fields, and according to the Matsubara formalism the boundary conditions over the fields are those consistent with the so called KMS (Kubo, Martin and Schwinger) conditions, stating periodic (antiperiodic) conditions for bosons (fermions). Such a topological result has actually been generalized to treat different physical situations in which fields are confined in higher space dimensions, considering the Matsubara mechanism on a $R^{D-N} \times S^{1_{1}} \times S^{1_{2}} \ldots \times S^{1_{N}}$ topology, describing time (temperature) and space confinement [4, 5, 6]. Along the same lines, but considering the thermofield dynamics (TFD) formalism, which is an operator formalism equivalent to the Matsubara approach [7, 8, 9, 10, 11, 12, 13] for systems in equilibrium, a generalization of the Bogoliubov transformation has been introduced to deal with confined boson fields in space coordinates at finite temperature, being an effective tool to derive different aspects of the Casimir effect for the electromagnetic field confined between two plates [14]. In this context one interesting physical result is brought about: the Casimir effect is described by a process of condensation of the field, thus shedding a new light on the nature of the quantum vacuum and the origin of the Casimir effect. In this paper we extend this approach introduced for boson fields in Ref. [14] to fermion fields, and we apply it to the Casimir effect of a free massless fermionic field between two plates, in a wave-guide and in a box.

In the realm of the quantum field theory, the Casimir effect was first proposed as a result of the vacuum fluctuation of the electromagnetic field confined between two plates with separation $L$, defined by the Dirichlet boundary conditions. The effect was an attractive force between the plates given by the negative pressure $P=-\pi^{2} / 240 L^{4}$ (we use natural units: $\hbar=c=1$ ) [15]. Over the decades, the Casimir effect has been applied to different geometries, fields and physical conditions, enjoying a remarkable popularity 16, 17, 18, 19, 20, 21, 22, 23, 24, 25, 26, 27, 28, 29, 30, 31, 32, 33] and raising interest, in particular, in the context of microelectronics [34, 35] as a practical tool for switching devices. 
The effect of temperature was first studied by Lifshitz [36, 37] who presented an alternative derivation for the Casimir force, including an analysis of the dielectric nature of the material between the plates. Actually, the effect of temperature on the interaction between the conducting parallel plates may be significant for separations greater than $3 \mu \mathrm{m}$ [38, 39, 40, 41, 42, 43]. For this physical set-up of plates, the full analysis of the thermal energy-momentum tensor of the electromagnetic field was carried out by Brown and Maclay [44], performing the calculation of the Casimir free-energy by using the Green's function (the local formulation) written in a conformally invariant way [23, 45, 46]. One of our proposal here is to derive the fermionic counterpart of the Brown and Maclay's formula, but for a more general situation of confinement: we consider not only the two plates, but also the case of confinement within a $d$-dimensional box.

Casimir effect for a fermionic field is of interest in considering, for instance, the structure of proton in particle physics; thus its physical appeal. In particular, in the phenomenological MIT bag model [47], quarks are confined in a small space region in such a way that there is no fermionic current outside that region. The fermion field then fulfills the so-called bag model boundary condition. The Casimir effect in such a small region, of the order of $1.0 \mathrm{fm}$, is important to define the process of deconfinement in heavy ion collisions at Relativistic Heavy Ion Collider (RHIC), giving rise to the quark-gluon plasma [48]. The gluon field contribution for the Casimir effect is, up to the color quantum numbers, the same as for the electromagnetic field. For the quark field, the problem has been often addressed by considering the case of two parallel plates [49, 50, 51, 52, 53, 54].

As first demonstrated by Johnson [55], for plates, the fermionic Casimir force is attractive as in the case of the electromagnetic field. On the other hand, depending on the geometry of confinement, the nature of the Casimir force can change. This is the case, for instance, for a spherical cavity and of the Casimir-Boyer model, using mixed boundary conditions for the electromagnetic field, such that the force is repulsive [56, 57, 58, 59, 60, 61]. Therefore, the analysis considering fermions in a wave-guide (confinement in two-dimensions) and in a 3-dimensional box (confinement in 3-dimensions) may be of interest. We avoid here the approach based on the sum of quantum modes, that was so important in the calculation of the Brown and Maclay. Using, alternately, the method developed earlier [14], we perform the calculation of the non-trivial problem of the Dirac field in a finite volume with the compactification in an $d$-dimensional box, at finite temperature. We hope then that this possibility for 
calculations may be useful in the general problem of fermions fulfilling boundary conditions, which are some times described by coupling with a static background field [62].

In order to proceed, we have to adapt the methodology of calculations discussed earlier 14], to encompass a generalized Bogoliubov transformation for fermion fields with the MIT bag model boundary condition; equivalent to an antiperiodic boundary condition [50]. This is presented in Section 2, where we derive the energy-momentum tensor for the fermion field at $T \neq 0$, resulting, as an example, in the Stefan-Boltzmann law. This is the usual calculation of TFD for fermions; however it can also be interpreted as a confinement in the time axis, in such a way that the field is under anti-periodic boundary conditions. Using then this methodology for a Euclidian geometry, we can envisage a space compactification. The usual expression of the Casimir effect is thus calculated, considering a proper modified Bogoliubov transformation which will describe the confinement in the z-axis. The familiarity with this kind of calculation for these already known results will suggest to us a general form for a Bogoliubov transformation to describe space compactification in arbitrary dimensions; a subject developed in Section 3. In Section 4, we consider the first application of the energy-momentum tensor derived in Section 3: we calculate the Casimir effect considering that the fermionic field is confined between two plates at finite temperature. As a particular consequence, we reach the expression derived in Ref. [49] for the Casimir energy, following the Matsubara formalism. In Section 5 we address the problem of the Casimir effect for a rectangular wave guide, and some particular cases are analyzed, resulting, for instance, in an attractive or repulsive Casimir force on opposite faces depending on the relative magnitude of the edges of the wave-guide transversal section. In Section 6 we consider the field confined in a parallelepiped box, showing similar aspects as those appearing in wage-guides. We also determine the temperature at which the Casimir pressure in a cubic box changes sign, estimating its value when the edge of the cube is of the order of the proton diameter. In Section 7 some concluding remarks are presented.

\section{TFD REVISITED}

The general approach to be used here can be addressed through the following prescription, taken as a generalization of the TFD formalism [7, 8, 14] . Given an arbitrary set of operators, say $\mathcal{V}$, with elements denoted by $A_{i}, i=1, \ldots, n$, there exists a mapping describing a doubling 
in the degrees of freedom defined by $\tau: \mathcal{V} \rightarrow \mathcal{V}$, denoted by $\tau A \tau^{-1}=\widetilde{A}$, satisfying the following conditions

$$
\begin{aligned}
\left(A_{i} A_{j}\right) & =\widetilde{A}_{i} \widetilde{A}_{j}, \\
\left(c A_{i}+A_{j}\right) & =c^{*} \widetilde{A}_{i}+\widetilde{A}_{j}, \\
\left(A_{i}^{\dagger}\right) & =\left(\widetilde{A}_{i}\right)^{\dagger}, \\
\left(\widetilde{A}_{i}\right) & =A_{i} .
\end{aligned}
$$

These properties are called the tilde (or dual) conjugation rules. The doubled Hilbert space has a new vacuum denoted by $|0, \widetilde{0}\rangle$. Consider $\alpha$ a $c$-number associated with macroscopic parameters of the system such as temperature, $\beta=1 / T$ (we shall show later that $\alpha$ may also stand for parameters describing the space confinement of fields as the distance between plates). The quasi-particles in TFD are introduced by a Bogoliubov transformation given by

$$
B(\alpha)=\left(\begin{array}{rr}
u(\alpha) & -v(\alpha) \\
v(\alpha) & u(\alpha)
\end{array}\right)
$$

with $u^{2}(\alpha)+v^{2}(\alpha)=1$.

For an arbitrary operator in $\mathcal{V}$, we use the doublet notation [7]

$$
\begin{aligned}
\left(A^{a}(\alpha)\right) & =\left(\begin{array}{c}
A(\alpha) \\
\widetilde{A}^{\dagger}(\alpha)
\end{array}\right)=B(\alpha)\left(\begin{array}{c}
A \\
\widetilde{A}^{\dagger}
\end{array}\right), \\
\left(A^{a}(\alpha)\right)^{\dagger} & =\left(A^{\dagger}(\alpha), \widetilde{A}(\alpha)\right) .
\end{aligned}
$$

This notation is useful to calculate the propagator for the confined field.

Here we are concerned with the energy-momentum tensor for a massless fermionic field given by 63 ]

$$
\begin{aligned}
T^{\mu \nu}(x) & =\left.\left\langle 0\left|i \bar{\psi}\left(x^{\prime}\right) \gamma^{\mu} \partial^{\nu} \psi(x)\right| 0\right\rangle\right|_{x^{\prime} \rightarrow x} \\
& =\left.\gamma^{\mu} \partial^{\nu} S\left(x-x^{\prime}\right)\right|_{x^{\prime} \rightarrow x} \\
& =-\left.4 i \partial^{\mu} \partial^{\nu} G_{0}\left(x-x^{\prime}\right)\right|_{x^{\prime} \rightarrow x}
\end{aligned}
$$

where $S\left(x-x^{\prime}\right)=-i\left\langle 0\left|T\left[\psi(x) \bar{\psi}\left(x^{\prime}\right)\right]\right| 0\right\rangle$ and $G_{0}\left(x-x^{\prime}\right)$ is the propagator of the free massless bosonic field. In the 4-dimensional space-time (to which we shall restrict most of our analysis) 
one has

$$
\begin{aligned}
G_{0}(x) & =\frac{-1}{(2 \pi)^{4}} \int d^{4} k e^{-i k \cdot x} G_{0}(k) \\
& =\frac{-i}{(2 \pi)^{2}} \frac{1}{x^{2}-i \varepsilon},
\end{aligned}
$$

where

$$
G_{0}(k)=\frac{1}{k^{2}+i \varepsilon}
$$

and the Minkowski metric has the signature $(+---)$. With $T^{\mu \nu}(x)$, we can introduce the confined ( $\alpha$-dependent) energy-momentum tensor $\mathcal{T}^{\mu \nu(a b)}(x ; \alpha)$ defined by

$$
\mathcal{T}^{\mu \nu(a b)}(x ; \alpha)=\left\langle T^{\mu \nu(a b)}(x ; \alpha)\right\rangle-\left\langle T^{\mu \nu(a b)}(x)\right\rangle,
$$

where $T^{\mu \nu(a b)}(x ; \alpha)$ is a function of the field operators $\psi(x ; \alpha), \widetilde{\psi}(x ; \alpha)$, according to Eq. (6), and $\langle\cdots\rangle=\langle 0, \widetilde{0}|\cdots| 0, \widetilde{0}\rangle$. Let us then work out this tensor.

Considering the TFD prescription [7, 64], we have

$$
S^{(a b)}\left(x-x^{\prime}\right)=\left(\begin{array}{cc}
S\left(x-x^{\prime}\right) & 0 \\
0 & \widetilde{S}\left(x-x^{\prime}\right)
\end{array}\right),
$$

with $\widetilde{S}\left(x-x^{\prime}\right)=-S^{*}\left(x^{\prime}-x\right)$. As a result, from Eq. (91), we have

$$
\mathcal{T}^{\mu \nu(a b)}(x ; \alpha)=-4 i \partial^{\mu} \partial^{\nu}\left[G_{0}^{(a b)}\left(x-x^{\prime} ; \alpha\right)-G_{0}^{(a b)}\left(x-x^{\prime}\right)\right]_{x^{\prime} \rightarrow x},
$$

corresponding to a change in Eq. (8),$S^{(a b)}\left(x-x^{\prime}\right)$ by $S\left(x-x^{\prime}\right)$. The Green's functions in Eq. (10) are given by

$$
G_{0}^{(a b)}\left(x-x^{\prime}\right)=\frac{-1}{(2 \pi)^{4}} \int d^{4} k G_{0}^{(a b)}(k) e^{-i k \cdot\left(x-x^{\prime}\right)},
$$

where

$$
G_{0}^{(a b)}(k)=\left(\begin{array}{cc}
G_{0}(k) & 0 \\
0 & G_{0}^{*}(k)
\end{array}\right)
$$

and the $\alpha$-counterpart is

$$
G_{0}^{(a b)}\left(x-x^{\prime} ; \alpha\right)=\frac{-1}{(2 \pi)^{4}} \int d^{4} k G_{0}^{(a b)}(k ; \alpha) e^{-i k \cdot\left(x-x^{\prime}\right)},
$$

with

$$
G_{0}^{(a b)}(k ; \alpha)=B_{k}^{-1(a c)}(\alpha) G_{0}^{(c d)}(k) B_{k}^{(d b)}(\alpha),
$$


where $B_{k}^{(a b)}(\alpha)$ is the Bogoliubov transformations given in Eq. (5). Explicitly, the components of $G_{0}^{(a b)}(k ; \alpha)$ are given by

$$
\begin{aligned}
& G_{0}^{11}(k ; \alpha)=G_{0}(k)+v_{k}^{2}(\alpha)\left[G_{0}^{*}(k)-G_{0}(k)\right], \\
& G_{0}^{12}(k ; \alpha)=G_{0}^{21}(k ; \alpha)=v_{k}(\alpha)\left[1-v_{k}^{2}(\alpha)\right]^{1 / 2}\left[G_{0}^{*}(k)-G_{0}(k)\right], \\
& G_{0}^{22}(k ; \alpha)=G_{0}^{*}(k)+v_{k}^{2}(\alpha)\left[G_{0}(k)-G_{0}^{*}(k)\right] .
\end{aligned}
$$

The physical quantities are derived from the component $G_{0}^{11}(k ; \alpha)$. Therefore, the physical $\alpha$-tensor is given by the component $\mathcal{T}^{\mu \nu(11)}(x ; \alpha)$.

Let us consider a simple situation in which $\alpha \equiv \beta=1 / T$. In this case $v_{k}(\beta)$ is defined through the fermion number distribution, that is

$$
v_{k}(\beta)=\frac{e^{-\beta k_{0} / 2}}{\left[1+e^{-\beta k_{0}}\right]^{1 / 2}}
$$

Observe that we can write

$$
v_{k}^{2}(\beta)=\sum_{l=1}^{\infty}(-1)^{l+1} e^{-\beta k_{0} l}
$$

leading to the thermal Green's function,

$$
G_{0}^{11}(k ; \beta)=G_{0}(k)+\sum_{l=1}^{\infty}(-1)^{l+1} e^{-\beta k_{0} l}\left[G_{0}^{*}(k)-G_{0}(k)\right] .
$$

Using this result in Eq. (11) we derive

$$
G_{0}^{11}\left(x-x^{\prime} ; \beta\right)=G_{0}\left(x-x^{\prime}\right)+\sum_{l=1}^{\infty}(-1)^{l+1}\left[G_{0}^{*}\left(x^{\prime}-x+i \beta l \widehat{n}_{0}\right)-G_{0}\left(x-x^{\prime}-i \beta l \widehat{n}_{0}\right)\right],
$$

where $\widehat{n}_{0}=(1,0,0,0)$ is a time-like vector. Therefore, from Eq. (10), we find

$$
\mathcal{T}^{\mu \nu(11)}(\beta)=-\left.4 i \sum_{l=1}^{\infty}(-1)^{l+1} \partial^{\mu} \partial^{\nu}\left[G_{0}^{*}\left(x^{\prime}-x+i \beta l \widehat{n}_{0}\right)-G_{0}\left(x-x^{\prime}-i \beta l \widehat{n}_{0}\right)\right]\right|_{x^{\prime} \rightarrow x} .
$$

Performing the covariant derivatives, this expression reads

$$
\mathcal{T}^{\mu \nu(11)}(\beta)=\frac{4}{\pi^{2}} \sum_{l=1}^{\infty}(-1)^{l}\left[\frac{g^{\mu \nu}-4 \widehat{n}_{0}^{\mu} \widehat{n}_{0}^{v}}{(\beta l)^{4}}\right] .
$$

Well known results for thermal fermionic fields can be derived from this tensor. For instance, the internal energy is given by $E(T)=\mathcal{T}^{00(11)}(\beta)$, that is,

$$
E(T)=\frac{7 \pi^{2}}{60} T^{4}
$$


where we have used the Riemann alternating zeta-function

$$
\varsigma(4)=\sum_{l=1}^{\infty}(-1)^{l+1} \frac{1}{l^{4}}=\frac{7 \pi^{4}}{720} .
$$

As another application, we derive the Casimir effect at zero temperature, by following the above calculations. For parallel plates perpendicular to the $x^{3}$-direction and separated by a distance $L$, instead of Eq. (12), we take $\alpha=i 2 L$, write

$$
v_{k}^{2}(L)=\sum_{l=1}^{\infty}(-1)^{l+1} e^{-i 2 L k_{3} l}
$$

and use $\widehat{n}_{3}=(0,0,0,1)$, a space-like vector. As a consequence, we derive

$$
\mathcal{T}^{\mu \nu(11)}(L)=\frac{4}{\pi^{2}} \sum_{l=1}^{\infty}(-1)^{l}\left[\frac{g^{\mu \nu}+4 \widehat{n}_{3}^{\mu} \widehat{n}_{3}^{\nu}}{(2 L l)^{4}}\right],
$$

resulting in the Casimir energy and the Casimir pressure given, respectively, by

$$
\begin{aligned}
& E_{c}(L)=\mathcal{T}^{00(11)}(L)=-\frac{7 \pi^{2}}{2880} \frac{1}{L^{4}} \\
& P_{c}(L)=\mathcal{T}^{33(11)}(L)=-\frac{7 \pi^{2}}{960} \frac{1}{L^{4}} .
\end{aligned}
$$

Notice that the choice of $\alpha$ as a pure imaginary number is required in order to obtain the spatial confinement, while the factor 2 is needed to ensure antiperiodic boundary conditions on the propagator. In the next section we extend this procedure to the situation where

multiple compactification of (imaginary) time and spatial coordinates are simultaneously implemented.

\section{COMPACTIFICATION IN HIGHER DIMENSIONS}

In this section we calculate the Casimir effect for massless fermions within a $d$-dimensional (space) "box" at finite temperature. For sake of generality, we will consider the $(1+N)$ dimensional Minkowski space. We then proceed by extending the results for the Casimir effect at zero and finite temperature derived in the last section. Supported by these calculations, we consider a generalization of $v(\alpha)$ (as given in Eq. (12)) by taking $\alpha=$ 
$\left(\alpha_{0}, \alpha_{1}, \alpha_{2}, \ldots, \alpha_{N}\right)$ and writing

$$
\begin{aligned}
v_{k}^{2}(\alpha)= & \sum_{j=0}^{N} \sum_{l_{j}=1}^{\infty}(-1)^{l_{j}+1} f\left(\alpha_{j}\right) \exp \left\{i \alpha_{j} l_{j} k_{j}\right\} \\
& +\sum_{j<r=0}^{N} \sum_{l_{j}, l_{r}=1}^{\infty}(-1)^{l_{j}+l_{r}+2} f\left(\alpha_{j}\right) f\left(\alpha_{r}\right) \exp \left\{i \alpha_{j} l_{j} k_{j}+i \alpha_{r} l_{r} k_{r}\right\}+\cdots \\
& +\sum_{l_{0}, l_{1}, \ldots, l_{N}=1}^{\infty}(-1)^{l_{0}+l_{1}+\ldots+l_{N}+N+1} f\left(\alpha_{0}\right) f\left(\alpha_{1}\right) \cdots f\left(\alpha_{N}\right) \exp \left\{i \sum_{i=0}^{N} \alpha_{i} l_{i} k_{i}\right\},
\end{aligned}
$$

where $f\left(\alpha_{j}\right)=0$ for $\alpha_{j}=0$ and $f\left(\alpha_{j}\right)=1$ otherwise. This expression leads, within the TFD formalism, to the simultaneous compactification of any $p(1 \leq p \leq N+1)$ dimensions corresponding to the non null parameters $\alpha_{j}$, with $\alpha_{0}$ corresponding to the time coordinate and $\alpha_{n}(n=1, \ldots, N)$ referring to the spatial ones. As we will see later, this expression is an immediate generalization from the results of the Casimir effect for two parallel plates at finite temperature derived by other methods.

A more compact expression for $v_{k}^{2}(\alpha)$ is

$$
v_{k}^{2}(\alpha)=\sum_{s=1}^{N+1} \sum_{\left\{\sigma_{s}\right\}}\left(\prod_{n=1}^{s} f\left(\alpha_{\sigma_{n}}\right)\right) \sum_{l_{\sigma_{1}}, \ldots, l_{\sigma_{s}}=1}^{\infty}(-1)^{s+\sum_{r=1}^{s} l_{\sigma_{r}}} \exp \left\{i \sum_{j=1}^{s} \alpha_{\sigma_{j}} l_{\sigma_{j}} k_{\sigma_{j}}\right\}
$$

where $\left\{\sigma_{s}\right\}$ denotes the set of all combinations with $s$ elements, $\left\{\sigma_{1}, \sigma_{2}, \ldots \sigma_{s}\right\}$, of the first $N+1$ natural numbers $\{0,1,2, \ldots, N\}$, that is all subsets containing $s$ elements, which we choose to write in an ordered form with $\sigma_{1}<\sigma_{2}<\cdots<\sigma_{s}$. Using this $v_{k}^{2}(\alpha)$ and generalizing the procedure delineated in Section 2, the (11)-component of the $\alpha$-dependent Green's function in momentum space becomes

$$
\begin{aligned}
G_{0}^{11}(k ; \alpha)= & G_{0}(k)+\sum_{s=1}^{N+1} \sum_{\left\{\sigma_{s}\right\}}\left(\prod_{n=1}^{s} f\left(\alpha_{\sigma_{n}}\right)\right) \\
& \times \sum_{l_{\sigma_{1}}, \ldots, l_{\sigma_{s}}=1}^{\infty}(-1)^{s+\sum_{r=1}^{s} l_{\sigma_{r}}} \exp \left\{i \sum_{j=1}^{s} \alpha_{\sigma_{j}} l_{\sigma_{j}} k_{\sigma_{j}}\right\}\left[G_{0}^{*}(k)-G_{0}(k)\right] .
\end{aligned}
$$

Taking the inverse Fourier transform of this expression and defining the vectors $\widehat{n}_{0}=$ $(1,0,0,0, \ldots), \widehat{n}_{1}=(0,1,0,0, \ldots), \ldots, \widehat{n}_{N}=(0,0,0, \ldots, 1)$ in the $(1+N)$-dimensional Minkowski 
space, Eq. (10) leads to

$$
\begin{aligned}
\mathcal{T}^{\mu \nu(11)}(\alpha)= & -4 i \sum_{s=1}^{N+1} \sum_{\left\{\sigma_{s}\right\}}\left(\prod_{n=1}^{s} f\left(\alpha_{\sigma_{n}}\right)\right) \sum_{l_{\sigma_{1}}, \ldots, l_{\sigma_{s}}=1}^{\infty}(-1)^{s+\sum_{r=1}^{s} l_{\sigma_{r}}} \\
& \times\left.\partial^{\mu} \partial^{\nu}\left[G_{0}^{*}\left(x^{\prime}-x+\sum_{j=1}^{s} \xi_{\sigma_{j}} \alpha_{\sigma_{j}} l_{\sigma_{j}} \widehat{n}_{\sigma_{j}}\right)-G_{0}\left(x-x^{\prime}-\sum_{j=1}^{s} \xi_{\sigma_{j}} \alpha_{\sigma_{j}} l_{\sigma_{j}} \widehat{n}_{\sigma_{j}}\right)\right]\right|_{x^{\prime} \rightarrow x},
\end{aligned}
$$

where $\xi_{\sigma_{j}}=+1$, if $\sigma_{j}=0$, and $\xi_{\sigma_{j}}=-1$ for $\sigma_{j}=1,2, \ldots, N$.

As we have noticed in Section 2, to get the physical situations of finite temperature and spatial confinement, $\alpha_{0}$ has to be taken as a positive real number while $\alpha_{n}$, for $n=1,2, \ldots, N$,

must be pure imaginary of the form $i 2 L_{n}$; in these cases, one finds that $\alpha_{j}^{* 2}=\alpha_{j}^{2}$. Considering such choices for the parameters $\alpha_{j}$ and using the explicit form of $G_{0}(x)$ for the 4-dimensional space-time (corresponding to $N=3$ ), we obtain

$$
\begin{aligned}
\mathcal{T}^{\mu \nu(11)}(\alpha)= & -\frac{4}{\pi^{2}} \sum_{s=1}^{4} \sum_{\left\{\sigma_{s}\right\}}\left(\prod_{n=1}^{s} f\left(\alpha_{\sigma_{n}}\right)\right) \sum_{l_{\sigma_{1}}, \ldots, l_{\sigma_{s}}=1}^{\infty}(-1)^{s+\sum_{r=1}^{s} l_{\sigma_{r}}} \\
& \times \frac{1}{\left[\sum_{j=1}^{s} \xi_{\sigma_{j}}\left(\alpha_{\sigma_{j}} l_{\sigma_{j}}\right)^{2}\right]^{2}}\left[g^{\mu \nu}-\frac{2 \sum_{j, r=1}^{s}\left(1+\xi_{\sigma_{j}} \xi_{\sigma_{r}}\right)\left(\alpha_{\sigma_{j}} l_{\sigma_{j}}\right)\left(\alpha_{\sigma_{r}} l_{\sigma_{r}}\right) \widehat{n}_{\sigma_{j}}^{\mu} \widehat{n}_{\sigma_{r}}^{\nu}}{\sum_{j=1}^{s} \xi_{\sigma_{j}}\left(\alpha_{\sigma_{j}} l_{\sigma_{j}}\right)^{2}}\right] .
\end{aligned}
$$

Notice that the results given by Eqs. (13) and (17) are particular cases of the energymomentum tensor given by Eq. (23) , corresponding to $\alpha=(\beta, 0,0,0)$ and $\alpha=(0,0,0, i 2 L)$ respectively. Another important aspect is that $\mathcal{T}^{\mu \nu(11)}(\alpha)$ is traceless, as it should be. In order to obtain the physical meaning of $\mathcal{T}^{\mu \nu(11)}(\alpha)$, we have to analyze particular situations. Thus we rederive first some known (but not trivial) results considering $N=3$. It is important to notice that Eq. (21) is the proper generalization of the Bogoliubov transformation, compatible with the generalizations of the Matsubara formalism, for the case of fermions.

\section{CASIMIR EFFECT FOR TWO PLATES}

The situation of two parallel plates at zero temperature has already been analyzed in Section 2; for this case, taking $\alpha=(0,0,0, i 2 L)$, Eq. (23) reduces to Eq. (17) and one recovers the standard Casimir effect. Let us then consider the case of two parallel plates at finite temperature. In this case, both time and space confinement need to be included; this 
is done by taking $\alpha=(\beta, 0,0, i 2 L)$ in Eq. (23), where as before $\beta^{-1}=T$ is the temperature and $L$ is the distance between the plates perpendicular to the $x^{3}$-axis. Therefore we find,

$$
\begin{aligned}
\mathcal{T}^{\mu \nu(11)}(\beta, L)= & \frac{4}{\pi^{2}}\left\{\sum_{l_{0}=1}^{\infty}(-1)^{l_{0}} \frac{\left[g^{\mu \nu}-4 \widehat{n}_{0}^{\mu} \widehat{n}_{0}^{\nu}\right]}{\left(\beta l_{0}\right)^{4}}+\sum_{l_{3}=1}^{\infty}(-1)^{l_{3}} \frac{\left[g^{\mu \nu}+4 \widehat{n}_{3}^{\mu} \widehat{n}_{3}^{\nu}\right]}{\left(2 L l_{3}\right)^{4}}\right. \\
& \left.-\sum_{l_{0}, l_{3}=1}^{\infty}(-1)^{l_{0}+l_{3}} \frac{\left(\beta l_{0}\right)^{2}\left[g^{\mu \nu}-4 \widehat{n}_{0}^{\mu} \widehat{n}_{0}^{\nu}\right]+\left(2 L l_{3}\right)^{2}\left[g^{\mu \nu}+4 \widehat{n}_{3}^{\mu} \widehat{n}_{3}^{\nu}\right]}{\left[\left(\beta l_{0}\right)^{2}+\left(2 L l_{3}\right)^{2}\right]^{3}}\right\} .
\end{aligned}
$$

Using the summation (15), the Casimir energy $E_{c}(\beta, L)=\mathcal{T}^{00(11)}(\beta, L)$ is given by

$$
E_{c}(\beta, L)=\frac{7 \pi^{2}}{60} \frac{1}{\beta^{4}}-\frac{7 \pi^{2}}{2880} \frac{1}{L^{4}}-\frac{4}{\pi^{2}} \sum_{l_{0}, l_{3}=1}^{\infty}(-1)^{l_{0}+l_{3}} \frac{3\left(\beta l_{0}\right)^{2}-\left(2 L l_{3}\right)^{2}}{\left[\left(\beta l_{0}\right)^{2}+\left(2 L l_{3}\right)^{2}\right]^{3}}
$$

Taking the limit $L \rightarrow \infty$, this energy reduces to the Stefan-Boltzmann term given in Eq. (14), while making $\beta \rightarrow \infty$ one regains the Casimir effect for two plates at zero temperature presented in Eq. (18). The third term, which stands for the correction of temperature, remains finite as $\beta \rightarrow 0$ and so, as expected, the high temperature limit is dominated by the positive contribution of the Stefan-Boltzmann term.

The Casimir pressure, $P_{c}(\beta, L)=\mathcal{T}^{33(11)}(\beta, L)$, can be similarly obtained as

$$
P_{c}(\beta, L)=\frac{7 \pi^{2}}{180} \frac{1}{\beta^{4}}-\frac{7 \pi^{2}}{960} \frac{1}{L^{4}}+\frac{4}{\pi^{2}} \sum_{l_{0}, l_{3}=1}^{\infty}(-1)^{l_{0}+l_{3}} \frac{\left(\beta l_{0}\right)^{2}-3\left(2 L l_{3}\right)^{2}}{\left[\left(\beta l_{0}\right)^{2}+\left(2 L l_{3}\right)^{2}\right]^{3}} .
$$

It is to be noticed that for low temperatures (large $\beta$ ) the pressure is negative but, as the temperature increases, a transition to positive values happens. It is possible to determine the "critical" curve of this transition, $\beta_{c}=\chi_{0} L$, by searching for the value of ratio $\chi=\beta / L$ for which the pressure vanishes; this value, $\chi_{0}$, is the solution of the transcendental equation

$$
\frac{7 \pi^{2}}{180} \frac{1}{\chi^{4}}-\frac{7 \pi^{2}}{960}+\frac{4}{\pi^{2}} \sum_{l, n=1}^{\infty}(-1)^{l+n} \frac{(\chi l)^{2}-3(2 n)^{2}}{\left[(\chi l)^{2}+(2 n)^{2}\right]^{3}}=0
$$

given, numerically, by $\chi_{0} \simeq 1.45000$.

These results, which reproduce known features about the fermionic Casimir effect for parallel plates obtained following the Matsubara formalism [49], suggest naturally, as we have pointed out before, the generalization of the Bogoliubov transformation in TFD with the expression given in Eq. (21). In the following sections we shall discuss situations in which the field is confined in more than one space directions. We start describing the Casimir effect for the massless fermion field in a rectangular wave-guide, that is, considering confinement in two space directions. Then we address this problem including the effect of temperature. 


\section{CASIMIR EFFECT FOR A WAVE-GUIDE}

The situation of a rectangular wave-guide is defined here by considering the confinement along the $x^{2}$ and the $x^{3}$ axis. Then the Casimir effect at zero temperature is obtained from Eq. (23) by taking $\alpha=\left(0,0, i 2 L_{2}, i 2 L_{3}\right)$, that is

$$
\begin{aligned}
\mathcal{T}^{\mu \nu(11)}\left(L_{2}, L_{3}\right)= & \frac{1}{4 \pi^{2}}\left\{\sum_{l_{2}=1}^{\infty}(-1)^{l_{2}} \frac{\left[g^{\mu \nu}+4 \widehat{n}_{2}^{\mu} \widehat{n}_{2}^{\nu}\right]}{\left(L_{2} l_{2}\right)^{4}}+\sum_{l_{3}=1}^{\infty}(-1)^{l_{3}} \frac{\left[g^{\mu \nu}+4 \widehat{n}_{3}^{\mu} \widehat{n}_{3}^{\nu}\right]}{\left(L_{3} l_{3}\right)^{4}}\right. \\
& -\sum_{l_{2}, l_{3}=1}^{\infty}(-1)^{l_{2}+l_{3}}\left[\frac{\left(L_{2} l_{2}\right)^{2}\left[g^{\mu \nu}+4 \widehat{n}_{2}^{\mu} \widehat{n}_{2}^{\nu}\right]+\left(L_{3} l_{3}\right)^{2}\left[g^{\mu \nu}+4 \widehat{n}_{3}^{\mu} \widehat{n}_{3}^{\nu}\right]}{\left[\left(L_{2} l_{2}\right)^{2}+\left(L_{3} l_{3}\right)^{2}\right]^{3}}\right. \\
& \left.\left.+\frac{4\left(L_{2} l_{2}\right)\left(L_{3} l_{3}\right)\left[\widehat{n}_{2}^{\mu} \widehat{n}_{3}^{\nu}+\widehat{n}_{3}^{\mu} \widehat{n}_{2}^{\nu}\right]}{\left[\left(L_{2} l_{2}\right)^{2}+\left(L_{3} l_{3}\right)^{2}\right]^{3}}\right]\right\} .
\end{aligned}
$$

Making use of Eq. (15), the Casimir energy, $E_{c}\left(L_{2}, L_{3}\right)=\mathcal{T}^{00(11)}\left(L_{2}, L_{3}\right)$, is given by

$$
E_{c}\left(L_{2}, L_{3}\right)=-\frac{7 \pi^{2}}{2880}\left(\frac{1}{L_{2}^{4}}+\frac{1}{L_{3}^{4}}\right)-\frac{1}{4 \pi^{2}} \sum_{l_{2}, l_{3}=1}^{\infty} \frac{(-1)^{l_{2}+l_{3}}}{\left[\left(L_{2} l_{2}\right)^{2}+\left(L_{3} l_{3}\right)^{2}\right]^{2}},
$$

while the Casimir pressure, $P_{c}\left(L_{2}, L_{3}\right)=\mathcal{T}^{33(11)}\left(L_{2}, L_{3}\right)$, reads

$$
P_{c}\left(L_{2}, L_{3}\right)=-\frac{7 \pi^{2}}{2880}\left(\frac{3}{L_{3}^{4}}-\frac{1}{L_{2}^{4}}\right)+\frac{1}{4 \pi^{2}} \sum_{l_{2}, l_{3}=1}^{\infty}(-1)^{l_{2}+l_{3}} \frac{\left(L_{2} l_{2}\right)^{2}-3\left(L_{3} l_{3}\right)^{2}}{\left[\left(L_{2} l_{2}\right)^{2}+\left(L_{3} l_{3}\right)^{2}\right]^{3}} .
$$

For a square wave-guide $\left(L_{2}=L_{3}=L\right)$, the Casimir energy and the Casimir pressure (in this case, $\mathcal{T}^{33(11)}=\mathcal{T}^{22(11)}$ ) reduce to

$$
\begin{aligned}
& E_{c}(L)=-\left(\frac{7 \pi^{2}}{1440}+\frac{\mathcal{C}_{2}}{4 \pi^{2}}\right) \frac{1}{L^{4}}, \\
& P_{c}(L)=-\left(\frac{7 \pi^{2}}{1440}+\frac{\mathcal{C}_{2}}{4 \pi^{2}}\right) \frac{1}{L^{4}},
\end{aligned}
$$

where the constant $\mathcal{C}_{2}$ is given by

$$
\mathcal{C}_{2}=\sum_{l, n=1}^{\infty} \frac{(-1)^{l+n}}{\left(l^{2}+n^{2}\right)^{2}} \simeq 0.19368
$$

We see that $E_{c}$ and $P_{c}$ for a square wave-guide behave, as functions of $L$, in the same way as in the case of two parallel plates, both being negative, but with the energy decreasing (increasing in absolute value) and the pressure increasing (smaller absolute value) in the wave-guide case as compared with the two plates situation. 
On the other hand, if the case $L_{2} \neq L_{3}$ is considered, although $E_{c}$ remains negative whatever the ratio $\xi=L_{3} / L_{2}$ is, it is clear from Eq. (29) that the sign of the Casimir pressure depends heavily on the relative magnitude of $L_{2}$ and $L_{3}$. In fact, a transition from negative to positive pressure is observed as $\xi$ is increased; this feature is presented in Figure 1 where we plot $P_{c}=\mathcal{T}^{33(11)}$ for some rectangular wave-guides (characterized by different values of $\xi)$ as a function of $L\left(=L_{2}\right)$. These plots indicate the existence of a specific value of the ratio $\xi, \xi_{0}$, for which the Casimir pressure vanishes identically. This value is the solution of the transcendental equation

$$
-\frac{7 \pi^{2}}{2880}\left(\frac{3}{\xi^{4}}-1\right)+\frac{1}{4 \pi^{2}} \sum_{l, n=1}^{\infty}(-1)^{l+n} \frac{l^{2}-3(\xi n)^{2}}{\left[l^{2}+(\xi n)^{2}\right]^{3}}=0
$$

which is given, numerically, by $\xi_{0} \simeq 1.37955$; all rectangular wave-guides with the ratio between $L_{3}$ and $L_{2}$ equal to $\xi_{0}$ have null Casimir pressure $P_{c}=\mathcal{T}^{33(11)}$.

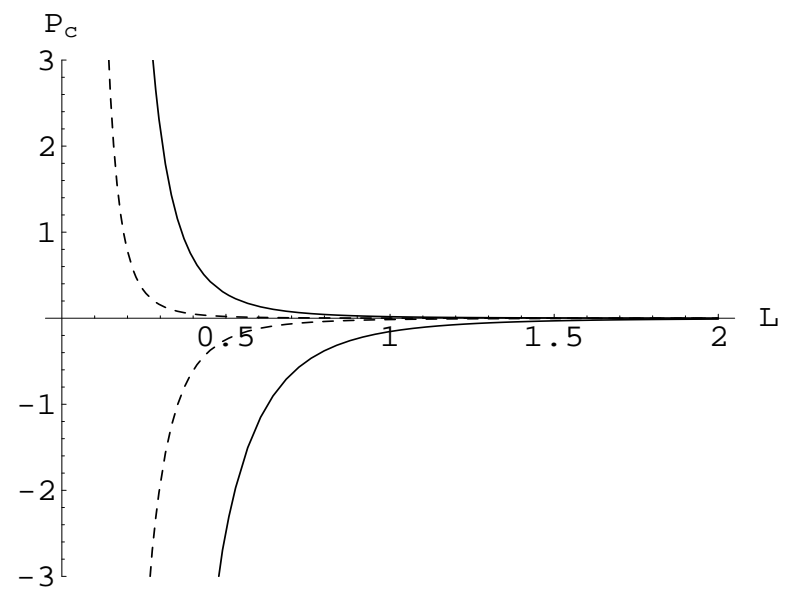

FIG. 1: The Casimir pressure, $P_{c}=\mathcal{T}^{33(11)}$, for some rectangular wave-guides, as a function of $L$ $\left(=L_{2}\right)$ : the full and the dashed lines below the horizontal axis correspond to $\xi=0.8$ and $\xi=1.2$, respectively; the dashed and the full lines above the horizontal axis refer to $\xi=1.4$ and $\xi=2.0$, respectively.

It is important to notice that the same kind of reasoning applies to $\mathcal{T}^{22(11)}\left(L_{2}, L_{3}\right)$, which can be obtained from $\mathcal{T}^{33(11)}$ by exchanging $L_{2} \leftrightarrow L_{3}$. So the force on the faces of the wage-guide perpendicular to the $x^{2}$-direction will also change from attractive to repulsive if the ratio $L_{2} / L_{3}=\xi^{-1}$ increases, passing by the value $\xi_{0}$. One sees that $\mathcal{T}^{33(11)}$ and $\mathcal{T}^{22(11)}$ will never be simultaneously positive; in fact, both are negative for $\xi_{0}^{-1}<\xi<\xi_{0}$ but they have opposite signs whenever $\xi>\xi_{0}$ or $\xi<\xi_{0}^{-1}$. It is also worth mentioning that, in a sense, 
this transition is similar to the transition from negative to positive pressures for two plates as the temperature is increased; both come from the compactification of a second space-time coordinate.

We now incorporate the effect of temperature in the wage-guide. To do so, we consider Eq. (23) with $\alpha=\left(\beta, 0, i 2 L_{2}, i 2 L_{3}\right)$. Then the Casimir energy becomes

$$
\begin{aligned}
E_{c}\left(\beta, L_{2}, L_{3}\right)= & \frac{7 \pi^{2}}{60} \frac{1}{\beta^{4}}-\frac{7 \pi^{2}}{2880}\left(\frac{1}{L_{2}^{4}}+\frac{1}{L_{3}^{4}}\right)+\frac{4}{\pi^{2}} \sum_{l_{0}, l_{2}=1}^{\infty}(-1)^{l_{0}+l_{2}} \frac{3\left(\beta l_{0}\right)^{2}-\left(2 L_{2} l_{2}\right)^{2}}{\left[\left(\beta l_{0}\right)^{2}+\left(2 L_{2} l_{2}\right)^{2}\right]^{3}} \\
& +\frac{4}{\pi^{2}} \sum_{l_{0}, l_{3}=1}^{\infty}(-1)^{l_{0}+l_{3}} \frac{3\left(\beta l_{0}\right)^{2}-\left(2 L_{3} l_{3}\right)^{2}}{\left[\left(\beta l_{0}\right)^{2}+\left(2 L_{3} l_{3}\right)^{2}\right]^{3}}-\frac{1}{4 \pi^{2}} \sum_{l_{2}, l_{3}=1}^{\infty} \frac{(-1)^{l_{2}+l_{3}}}{\left[\left(L_{2} l_{2}\right)^{2}+\left(L_{3} l_{3}\right)^{2}\right]^{2}} \\
& -\frac{4}{\pi^{2}} \sum_{l_{0}, l_{2}, l_{3}=1}^{\infty}(-1)^{l_{0}+l_{2}+l_{3}} \frac{3\left(\beta l_{0}\right)^{2}-\left(2 L_{2} l_{2}\right)^{2}-\left(2 L_{3} l_{3}\right)^{2}}{\left[\left(\beta l_{0}\right)^{2}+\left(2 L_{2} l_{2}\right)^{2}+\left(2 L_{3} l_{3}\right)^{2}\right]^{3}}
\end{aligned}
$$

while the Casimir pressure, $P_{c}=\mathcal{T}^{33(11)}$, is given by

$$
\begin{aligned}
P_{c}\left(\beta, L_{2}, L_{3}\right)= & \frac{7 \pi^{2}}{180} \frac{1}{\beta^{4}}-\frac{7 \pi^{2}}{2880}\left(\frac{3}{L_{3}^{4}}-\frac{1}{L_{2}^{4}}\right)+\frac{4}{\pi^{2}} \sum_{l_{0}, l_{3}=1}^{\infty}(-1)^{l_{0}+l_{3}} \frac{\left(\beta l_{0}\right)^{2}-3\left(2 L_{3} l_{3}\right)^{2}}{\left[\left(\beta l_{0}\right)^{2}+\left(2 L_{3} l_{3}\right)^{2}\right]^{3}} \\
& +\frac{4}{\pi^{2}} \sum_{l_{0}, l_{2}=1}^{\infty} \frac{(-1)^{l_{0}+l_{2}}}{\left[\left(\beta l_{0}\right)^{2}+\left(2 L_{2} l_{2}\right)^{2}\right]^{2}}+\frac{1}{4 \pi^{2}} \sum_{l_{2}, l_{3}=1}^{\infty}(-1)^{l_{2}+l_{3}} \frac{\left(L_{2} l_{2}\right)^{2}-3\left(L_{3} l_{3}\right)^{2}}{\left[\left(L_{2} l_{2}\right)^{2}+\left(L_{3} l_{3}\right)^{2}\right]^{3}} \\
& -\frac{4}{\pi^{2}} \sum_{l_{0}, l_{2}, l_{3}=1}^{\infty}(-1)^{l_{0}+l_{2}+l_{3}} \frac{\left(\beta l_{0}\right)^{2}+\left(2 L_{2} l_{2}\right)^{2}-3\left(2 L_{3} l_{3}\right)^{2}}{\left[\left(\beta l_{0}\right)^{2}+\left(2 L_{2} l_{2}\right)^{2}+\left(2 L_{3} l_{3}\right)^{2}\right]^{3}} .
\end{aligned}
$$

For simplicity we concentrate on the problem of a square wave-guide at finite temperature. As in the two-plates case, the Casimir energy passes from negative to positive values with increasing temperature, as expected since the Stefan-Boltzmann term dominates all others as $\beta \rightarrow 0$. Let us then look at the Casimir pressure $P_{c}=\mathcal{T}^{33(11)}\left(=\mathcal{T}^{22(11)}\right)$. Taking $L_{2}=L_{3}=L$ and defining $\chi=\beta / L$, Eq. (33) can be written as

$$
P_{c}(\chi, L)=g(\chi) \frac{1}{L^{4}}
$$

where

$$
\begin{aligned}
g(\chi)= & \frac{7 \pi^{2}}{180} \frac{1}{\chi^{4}}-\frac{7 \pi^{2}}{1440}-\frac{\mathcal{C}_{2}}{2 \pi^{2}}+\frac{4}{\pi^{2}} \sum_{l, n=1}^{\infty}(-1)^{l+n} \frac{(\chi l)^{2}-3(2 n)^{2}}{\left[(\chi l)^{2}+(2 n)^{2}\right]^{3}} \\
& +\frac{4}{\pi^{2}} \sum_{l, n=1}^{\infty} \frac{(-1)^{l+n}}{\left[(\chi l)^{2}+(2 n)^{2}\right]^{2}}-\frac{4}{\pi^{2}} \sum_{l, n, r=1}^{\infty}(-1)^{l+n+r} \frac{(\chi l)^{2}+(2 n)^{2}-3(2 r)^{2}}{\left[(\chi l)^{2}+(2 n)^{2}+(2 r)^{2}\right]^{3}} .
\end{aligned}
$$

As in the case of two plates, the Casimir pressure in a square wage-guide passes from negative to positive values as the temperature increases, the transition point given by the value of 
$\chi, \chi_{0}$, such that $g\left(\chi_{0}\right)=0$. One finds (numerically) $\chi_{0} \simeq 1.60224$, and so write down the critical curve $\beta_{c}=\chi_{0} L$. In the general case, $L_{2} \neq L_{3}$, increasing the temperature tends to make all diagonal components of $\mathcal{T}^{\mu \nu(11)}$ positive.

In the next section we analyze the Casimir effect for a massless fermion field confined in a rectangular parallelepiped box, taking into account the effect of temperature.

\section{CASIMIR EFFECT FOR A BOX}

We now consider the field confined in a 3-dimensional closed box having the form of a rectangular parallelepiped with faces $L_{1}, L_{2}$ and $L_{3}$. At zero temperature, the physical energy-momentum tensor is obtained from Eq. (23) by taking $\alpha=\left(0, i 2 L_{1}, i 2 L_{2}, i 2 L_{3}\right)$. The Casimir energy is then given by

$$
\begin{aligned}
E_{c}\left(L_{1}, L_{2}, L_{3}\right)= & -\frac{7 \pi^{2}}{2880}\left(\frac{1}{L_{1}^{4}}+\frac{1}{L_{2}^{4}}+\frac{1}{L_{3}^{4}}\right)-\frac{1}{4 \pi^{2}} \sum_{l_{1}, l_{2}=1}^{\infty} \frac{(-1)^{l_{1}+l_{2}}}{\left[\left(L_{1} l_{1}\right)^{2}+\left(L_{2} l_{2}\right)^{2}\right]^{2}} \\
& -\frac{1}{4 \pi^{2}} \sum_{l_{1}, l_{3}=1}^{\infty} \frac{(-1)^{l_{1}+l_{3}}}{\left[\left(L_{1} l_{1}\right)^{2}+\left(L_{3} l_{3}\right)^{2}\right]^{2}}-\frac{1}{4 \pi^{2}} \sum_{l_{2}, l_{3}=1}^{\infty} \frac{(-1)^{l_{2}+l_{3}}}{\left[\left(L_{2} l_{2}\right)^{2}+\left(L_{3} l_{3}\right)^{2}\right]^{2}} \\
& +\frac{1}{4 \pi^{2}} \sum_{l_{1}, l_{2}, l_{3}=1}^{\infty} \frac{(-1)^{l_{1}+l_{2}+l_{3}}}{\left[\left(L_{1} l_{1}\right)^{2}+\left(L_{2} l_{2}\right)^{2}+\left(L_{3} l_{3}\right)^{2}\right]^{2}},
\end{aligned}
$$

and the Casimir pressure, $P_{c}=\mathcal{T}^{33(11)}$, reads

$$
\begin{aligned}
P_{c}\left(L_{1}, L_{2}, L_{3}\right)= & -\frac{7 \pi^{2}}{2880}\left(\frac{3}{L_{3}^{4}}-\frac{1}{L_{1}^{4}}-\frac{1}{L_{2}^{4}}\right)+\frac{1}{4 \pi^{2}} \sum_{l_{1}, l_{3}=1}^{\infty}(-1)^{l_{1}+l_{3}} \frac{\left(L_{1} l_{1}\right)^{2}-3\left(L_{3} l_{3}\right)^{2}}{\left[\left(L_{1} l_{1}\right)^{2}+\left(L_{3} l_{3}\right)^{2}\right]^{3}} \\
& +\frac{1}{4 \pi^{2}} \sum_{l_{1}, l_{2}=1}^{\infty} \frac{(-1)^{l_{1}+l_{2}}}{\left[\left(L_{1} l_{1}\right)^{2}+\left(L_{2} l_{2}\right)^{2}\right]^{2}}+\frac{1}{4 \pi^{2}} \sum_{l_{2}, l_{3}=1}^{\infty}(-1)^{l_{2}+l_{3}} \frac{\left(L_{2} l_{2}\right)^{2}-3\left(L_{3} l_{3}\right)^{2}}{\left[\left(L_{2} l_{2}\right)^{2}+\left(L_{3} l_{3}\right)^{2}\right]^{3}} \\
& -\frac{1}{4 \pi^{2}} \sum_{l_{1}, l_{2}, l_{3}=1}^{\infty}(-1)^{l_{1}+l_{2}+l_{3}} \frac{\left(L_{1} l_{1}\right)^{2}+\left(L_{2} l_{2}\right)^{2}-3\left(L_{3} l_{3}\right)^{2}}{\left[\left(L_{1} l_{1}\right)^{2}+\left(L_{2} l_{2}\right)^{2}+\left(L_{3} l_{3}\right)^{2}\right]^{3}} .
\end{aligned}
$$

For a cubic box $\left(L_{1}=L_{2}=L_{3}=L\right)$, the Casimir energy and the Casimir pressure (now one has $\mathcal{T}^{33(11)}=\mathcal{T}^{22(11)}=\mathcal{T}^{11(11)}$ ) become

$$
\begin{aligned}
& E_{c}(L)=-\left(\frac{7 \pi^{2}}{960}+\frac{3 \mathcal{C}_{2}-\mathcal{C}_{3}}{4 \pi^{2}}\right) \frac{1}{L^{4}}, \\
& P_{c}(L)=-\left(\frac{7 \pi^{2}}{2880}+\frac{3 \mathcal{C}_{2}-\mathcal{C}_{3}}{12 \pi^{2}}\right) \frac{1}{L^{4}},
\end{aligned}
$$


where the constant $\mathcal{C}_{3}$ is given by

$$
\mathcal{C}_{3}=\sum_{l, n, r=1}^{\infty} \frac{(-1)^{l+n+r}}{\left(l^{2}+n^{2}+r^{2}\right)^{2}} \simeq-0.06314 .
$$

One sees that both energy and pressure in cubic boxes behave similarly to the cases of two parallel plates and of square wave-guides; in Figure 2 we plot the Casimir pressure for all these symmetrical situations, for comparison. It is curious that, in the units we are using, the Casimir pressure is three times the energy for parallel plates, they are equal in a square wave-guide, while in a cubic box one has $P_{c}(L)=E_{c}(L) / 3$.

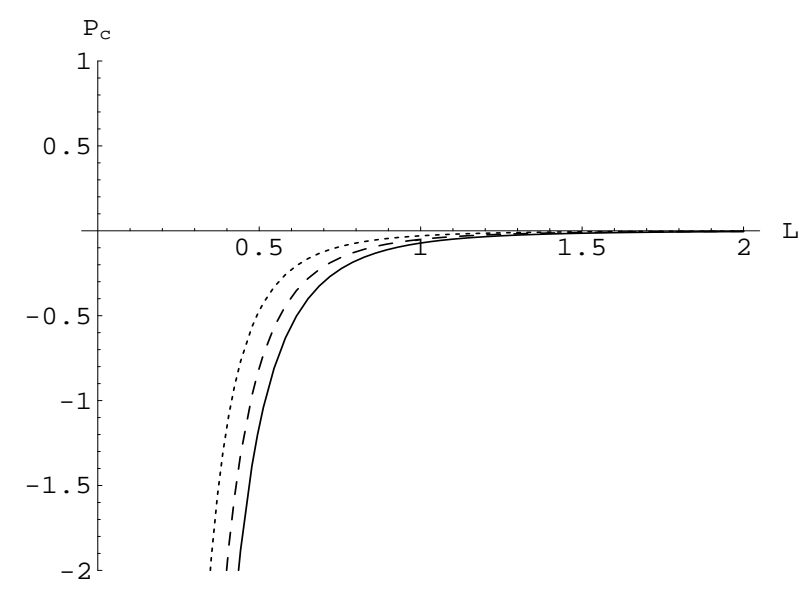

FIG. 2: The Casimir pressure, $P_{c}$, for two parallel plates separated by a distance $L$ (full line); for a square wave-guide with transversal section of edge $L$ (dashed line); and for a cubic box of edge $L$ (dotted line).

Changing the relative magnitude of the edges of the parallelepiped box leads to similar effects as in the case of the rectangular wave-guide. For example, taking $L_{1}=L_{2}=L$ and defining $\xi=L_{3} / L$, one can show that $P_{c}(L, \xi)=\mathcal{T}^{33(11)}(L, \xi)$ vanishes for $\xi=\xi_{0} \simeq 1.15274$, being negative for $\xi<\xi_{0}$ and positive for $\xi>\xi_{0}$. However, as in the case of the wave-guide, $\mathcal{T}^{22(11)}\left(=\mathcal{T}^{11(11)}\right.$ in the present situation) will be negative whenever $\mathcal{T}^{33(11)}>0$.

To treat the effect of temperature in the case of a box, all four coordinates in the Minkowski space have to be compactified by considering $\alpha=\left(\beta, i 2 L_{1}, 12 L_{2}, i 2 L_{3}\right)$ in Eq. (23). This amounts to the addition to Eqs. (36) and (37) terms involving $\beta$ and the distances $L_{j}$ like those appearing in Eqs. (32) and (33). In the simpler case of a cubic box, one finds that the Casimir pressure changes from negative to positive values when the ratio $\chi=\beta / L$ passes through the value $\chi_{0} \simeq 2.0323$. The critical curves, $\beta_{c}=\chi_{0} L$, for all symmetrical 
cases analyzed here (parallel plates, square wave-guide and cubic box) appear in Figure 3 . One notices that the behaviors of $T_{c} \times L$, in all three cases, are very similar one to each other, with $T_{c}$ scaling with the inverse fourth power of the relevant length $L$.

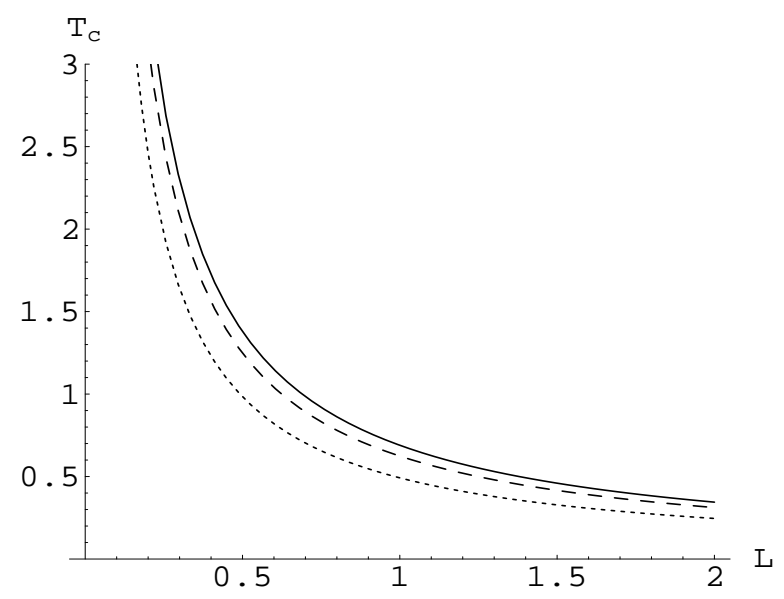

FIG. 3: Critical curves for the transitions from negative to positive Casimir pressure, induced by the temperature, for: (i) two parallel plates separated by a distance $L$ (full line); (ii) square waveguide of transversal section of edge $L$ (dashed line); (iii) cubic box of edge $L$ (dotted line). In all cases, the points below the curves correspond to $P_{c}<0$, while above them one has $P_{c}>0$.

On the basis of these results, we can estimate the temperature at which the Casimir pressure in a cubic box changes from negative to positive values considering the edge of the cube of the order of confining lengths for hadronic matter. Such an estimative provides a crude idea of the importance of Casimir effect in the deconfinement transition for hadrons. Taking $L \approx 1 \mathrm{fm}$, a length of the order of hadron radius, one finds $T_{c}=\left(\chi_{0} L\right)^{-1} \approx 100 \mathrm{MeV}$ from quark degrees of freedom considering only one flavor quark. For a real hadron there are two flavors, $u$ and $d$, each with 3 colors and an octet of gluons. In order to calculate the Casimir energy and pressure, the results for bosonic and fermionic fields have to be combined.

\section{CONCLUDING REMARKS}

In this paper we have presented a generalization of the Bogoliubov transformation in order to describe a massless fermion field compactified in a $d$-dimensional box at finite temperature. We write the (traceless) energy-momentum tensor from which we calculate and 
compare explicit expressions for the Casimir pressure and the Casimir energy, corresponding to different cases of confinement. The Casimir pressure at zero temperature for the cases of two parallel plates, square wave-guides and cubic boxes are negative (see Figure 2), imposing then an attractive force on all faces of the volume confining the fermionic field. On the other hand, for rectangular wave-guides and parallelepiped boxes the pressure on opposite faces can be made positive, representing a repulsive force, by appropriately choosing the ratios between the edges of the rectangle and between the edges of the cube. However, at zero temperature, one cannot make positive the pressure for all opposite faces of the wave-guide or of the box simultaneously; this can be achieved only by raising the temperature sufficiently. We have calculated the Casimir pressure at finite temperature for the cases of two parallel plates, square wave-guides and cubic boxes and determined the critical curves for these transitions from negative to positive pressures (see Figure 3). Since repulsive Casimir force can have a direct influence on the description of quark deconfinement, we have estimated this critical temperature for a cubic box of edge equal to $1 \mathrm{fm}$ (which is the order of magnitude of nuclear dimensions) obtaining $T_{c} \approx 100 \mathrm{MeV}$. It is important to emphasize that these results are only an estimate since these are based on the presence of one fermion flavor field in a cubic box. For realistic calculations, a spherical geometry with full account of the color and flavor degrees of freedom has to be considered.

It is important to stress the simplicity of these calculations, that is clear by comparing with the known results for the fermion Casimir effect; see for instance [22, 48, 49]. We have avoided usual procedures, the intricate method based on the sum of the quantum modes of the fields, satisfying some given boundary conditions. Indeed, instead of the sum of modes, we have used the generalized Bogoliubov transformation to define the Casimir effect which provides an elegant physical interpretation of the effect as a consequence of the condensation in the vacuum for the fermion field (in a similar fashion as was carried out for the case of bosons [14]). Taking advantages of these practical proposals, the method developed here can be useful for calculations involving other geometries with spherical or cylindrical symmetries. This analysis will be developed in more detail elsewhere.

Acknowledgments: This work was supported by CNPQ of Brazil, by NSERC of Canada, by the Technion-Haifa University Joint Research Fund, and by the Fund for Promotion of Sponsored Research at the Technion, Israel. One of us (AES) thanks M. Amato and 
T. M. Rocha-Filho for stimulating discussions and their interest in this work. One of us (JMCM) thanks the Theoretical Physics Institute and Vice-President (Research) for partial support during his stay at the University of Alberta.

[1] J. Polchinski, Commun. Math. Phys. 104 (1986) 37.

[2] J. J. Atick and E. Witten, Nucl. Phys. B 310 (1988) 291.

[3] L. Dolan and R. Jackiw, Phys. Rev. D 9 (1974) 3320.

[4] A. P. C. Malbouisson and J. M. C. Malbouisson, J. Phys. A: Math. Gen. 35 (2002) 2263.

[5] A. P. C. Malbouisson, J. M. C. Malbouisson and A. E. Santana, Nucl. Phys. B 631 (2002) 83.

[6] A. P. C. Malbouisson, J. M. C. Malbouisson, A. E. Santana, J. C. Silva , Phys. Lett. B 583 (2004) 373.

[7] H. Umezawa, Advanced Field Theory: Micro, Macro and Thermal Physics (AIP, New York, 1993).

[8] Y. Takahashi and H. Umezawa, Coll. Phenomena 2 (1975) 55 (Reprinted in Int. J. Mod. Phys. 10 (1996) 1755).

[9] H. Umezawa, H. Matsumoto and M. Tachiki, Thermofield Dynamics and Condensed States (North-Holland, Amsterdan, 1982).

[10] I. Ojima, Ann. Phys. (N. Y.) 137 (1981) 1.

[11] A. E. Santana, A. Matos Neto, J. D. M. Vianna and F. C. Khanna, Physica A 280 (2000) 405 .

[12] M. C. B. Abdala, A. L. Gadelha and I. V. Vancea, Phys. Rev. D 64 (2001) 086005.

[13] M. C. B. Abdala, A. L. Gadelha and I. V. Vancea, Int. J. Mod. Phys. A 18 (2003) 2109.

[14] J. C. da Silva, A. Matos Neto, F.C. Khanna and A. E. Santana, Phys. Rev. A 66 (2002) 052101.

[15] H. B. G. Casimir, Proc. Ned. Akad. Wet. B 51 (1948) 793.

[16] P. W. Milonni, The Quantum Vacuum (Academic, Boston, 1993).

[17] V. M. Mostepanenko and N.N. Trunov, The Casimir Effect and its Applications (Clarendon, Oxford, 1997).

[18] M. Bordag, U. Mohideed and V. M. Mostepanenko, New Developments in Casimir Effect, 
quant-ph/0106045, Phys. Rep. 353 (2001) 1.

[19] F. S. Levin and D.A. Micha (Eds.), Long Range Casimir Forces (Plenum, New York, 1993).

[20] C. Seife, Science 275 (1997) 158.

[21] T. H. Boyer, Am. J. Phys. 71 (2003) 990.

[22] K. A. Milton, The Casimir Effect: Physical Manifestations of Zero Point Energy, hep-th/9901011.

[23] G. Plunien, B. Müeller and W. Greiner, Phys. Rep. 134 (1986) 87.

[24] M. Bordag (Ed.), The Casimir Effect 50 Years Later (World Scientific, Singapore, 1999).

[25] G. Bressi, G. Carugno, R. Onofrio and G. Ruoso, Phys. Rev. Lett. 88 (2002) 041804.

[26] F. Caruso, N. P. Neto, B. F. Svaiter and N. F. Svaiter, Phys. Rev. D 43 (1991) 1300.

[27] F. Caruso, R. de Paola and N. F. Svaiter, Int. J. Mod. Phys. A 14 (1999) 2077.

[28] M. V. Cougo-Pinto, C. Farina and A. Tenório, Braz. J. Phys. 29 (1999) 371.

[29] D. T. Alves, C. Farina and A. C. Tort, Phys. Rev. A 63 (2000) 4102.

[30] S. K. Lamoreaux, Am. J. Phys. 67 (1999) 850.

[31] U. Mohideen and A. Roy, Phys. Rev. Lett. 81 (1998) 4549.

[32] O. Kenneth, I. Klich, A. Mann and M. Revzen, Phys. Rev. Lett. 89 (2002) 033001.

[33] T. Maruyama, K. Tsushima and A. Faessler, Nucl. Phys. A 537 (1992) 303.

[34] F. Serry, D. Walliser and G. J. Maclay, J. Appl. Phys. 84 (1998) 2501.

[35] E. Buks and M. L. Roukes, Phys. Rev. B 63 (2001) 033402.

[36] E. M. Lifshitz, Sov. Phys. JETP 2 (1956) 73.

[37] I. E. Dzyaloshinskii, E. M. Lifshitz and L. P. Pitaevskii, Adv. Phys.10 (1961) 165.

[38] J. Mehra, Physica, 37 (1967) 145.

[39] G. L. Klimchitskaya and V. M. Mostepanenko, Phys. Rev. A 63 (2001) 062108.

[40] M. Revzen, R. Opher, M. Opher and A. Mann, Europhys. Lett. 38 (1997) 245 (1997).

[41] M. Revzen, R. Opher, M. Opher and A. Mann, J. Phys. A: Math and Gen. 30 (1997) 7783.

[42] M. Revzen and A. Mann, Casimir Effect - The Classical Limit, quant-ph/9803059.

[43] J. Feinberg, A. Mann and M. Revzen, Ann. Phys.(NY) 288 (2001) 103.

[44] L. S. Brown and G. J. Maclay, Phys. Rev. 184 (1969) 1272.

[45] D. Robaschik, K. Scharnhorst and E. Wieczorek, Ann. Phys.(N.Y.) 174 (1987) 401.

[46] S. Tadaki and S. Takagi, Prog. Theor. Phys. 75 (1982) 262.

[47] A. Chodos, R. L. Jaffe, K. Johnson, C. B. Thorn and V. F. Weisskopf, Phys. Rev. D 9 (1974) 
3471.

[48] K. Saito, Z. Phys. C 50 (1991) 69.

[49] S. A. Gundersen and F. Ravndal, Ann. Phys. (N.Y.) 182 (1988) 90.

[50] C. A. Lütken and F. Ravndal, J. Phys. A: Math. Gen. 21 (1988) L793.

[51] C. A. Lütken and F. Ravndal, J. Phys. G: Nucl. Phys. 10 (1984) 123.

[52] F. Ravndal and D. Tollefsen, Phys. Rev. D 40 (1989) 4191.

[53] R. D. M. De Paola, R. B. Rodrigues and N. F. Svaiter, Mod. Phys. Lett. A 14 (1999) 2353.

[54] E. Elizalde, F. C. Santos and A. C. Tort, Int. J. Mod. Phys. A 18 (2003) 1761, hep-th/0206114.

[55] K. Johnson, Acta Phys. Pol. B 6 (1975) 865.

[56] L. A. Ferreira, A. H. Zimerman and J. R. Ruggiero, Casimir Effect for Closed Cavities with Conducting and Permeable Walls, Instituto de Física Teórica, UNESP, Preprint IFT-P-15/80.

[57] W. Lukosz, Physica 56 (1971) 109

[58] J. Ambj $\phi$ rn and S. Wolfram, Ann. Phys. (N.Y.) 147 (1983) 1.

[59] J. Ambjфrn and S. Wolfram, Ann. Phys. (N.Y.) 147 (1983) 33.

[60] T. H. Boyer, Phys. Rev. A 9 (1974) 2078.

[61] J. C. da Silva, A. Matos Neto, H. Queiroz Plácido, M. Revzen and A. E. Santana, Physica A $292(2001) 411$.

[62] P. Sundberg and R. L. Jaffe, Ann. Phys. (NY) 309 (2004) 442.

[63] C. Itzykson and J. B. Zuber, Quantum Field Theory (McGrow-Hill, New York, 1980).

[64] K. Soutome, Z. Phys. C 40 (1988) 479. 